\title{
Classification of stillbirths by relevant condition at death (ReCoDe): a cross sectional study at a rural tertiary care centre in Kerala, India
}

\author{
Ajini K. K.*, Radha K. R., Reena R.P.
}

Department of Obstetrics and Gynecology, Government Medical College, Thrissur, Kerala, India

Received: 05 January 2017

Accepted: 04 February 2017

*Correspondence:

Dr. Ajini K. K.,

E-mail: ajinivijoy@gmail.com

Copyright: $\odot$ the author(s), publisher and licensee Medip Academy. This is an open-access article distributed under the terms of the Creative Commons Attribution Non-Commercial License, which permits unrestricted non-commercial use, distribution, and reproduction in any medium, provided the original work is properly cited.

\section{ABSTRACT}

Background: Stillbirths account for a major proportion of perinatal deaths. The cause for a large number of stillbirths, remain unexplained. The ReCoDe system (classification of stillbirths by relevant condition at death) has helped to improve our understanding of the causes of stillbirths. We evaluated stillbirths and classified them by the ReCoDe system. The objective was to identify factors associated with stillbirths and to classify the causes of stillbirths using the ReCoDe system.

Methods: We recruited all women admitted to a tertiary care centre with a diagnosis of intrauterine fetal death after 22 weeks of gestation. Maternal sociodemographic characteristics and relevant antenatal and intrapartum investigations were studied. We examined the stillborn babies, placenta and umbilical cord and classified them according to the ReCoDe system.

Results: There were177 women with intrauterine fetal death admitted during the 2 year study period. The stillbirth rate was 38.56 per 1000 live births. We could explain the cause of stillbirth in $87.58 \%$ of cases by using the ReCoDe system. Intra uterine growth restriction (41.8\%) was the commonest cause followed by hypertensive disorders (27.68\%), congenital anomalies (15.81\%) and diabetes (14.68\%) in present study.

Conclusions: We found the ReCoDe system useful in classifying the cause of stillbirths in resource limited settings.

Keywords: IUGR, Perinatal mortality, ReCoDe, Stillbirths

\section{INTRODUCTION}

Stillbirth is one of the most devastating complications of pregnancy. The term stillbirth refers to the delivery of a fetus after 22 completed weeks of gestation, weighing $500 \mathrm{gms}$ or more with new born baby showing no signs of life after delivery. ${ }^{1}$ There are significant differences in the rate and causes of stillbirths in different parts of the world. Most (98\%) of these deaths occur in the developing world. This is influenced by a wide range of maternal, social and circumstantial factors. ${ }^{2}$

Perinatal mortality is often seen as a quality parameter for obstetric and neonatal care. Stillbirths are the leading cause of perinatal loss. The estimates of the clinical categories of stillbirth are often made after classifying the causes. The classification system helps clinicians and caregivers to understand what went wrong. This serves to guide changes in clinical practice so as to improve outcomes in future pregnancies. The new ReCoDe classification system was derived after a populationbased cohort study (1997-2003) in West Midlands Region (Perinatal Institute). ${ }^{3}$ The ReCoDe classification system identifies $85 \%$ of the conditions associated with stillbirth unlike other classifications systems like amended Aberdeen or extended Wigglesworth classifications which would identify only one third causes of stillbirths leaving most (66.2\%) unclassified. ${ }^{4}$ Many of the classification systems depend on extensive investigations and foetal autopsy. Most clinicians are 
hesitant to recommend perinatal autopsy and parents refuse to comply with it due to cultural and emotional factors.

ReCoDe is currently the only classification system specifically developed for classification of causes of stillbirths. $^{5}$ ReCoDe is a clinically based system appropriate for developing countries where only minimal investigations are possible. We evaluated all cases of stillbirths using ReCoDe classification system to find out the cause of fetal loss and studied factors associated with it.

\section{METHODS}

We used a cross sectional study design to evaluate the causes of stillbirths at the Department of Obstetrics and Gynecology, Government Medical College, Thrissur, Kerala, India from March 2014 to February 2016.The study protocol was accepted by the Institutional Review Board. Subjects were recruited after taking informed consent.

All pregnant women admitted to our department were screened to identify intrauterine fetal death after 22 weeks of gestation. All women with antepartum and intrapartum intra uterine fetal death were included. Women with a history of attempted late feticide or admitted after having delivered stillborns elsewhere and those not consenting to take part in the study were excluded. A detailed history was taken including the age, parity, socio economic status, body mass index, particulars of antenatal care, past and present medical and surgical history, including drug intake. Gestational age was confirmed by either reliable dates or a first trimester antenatal ultra sound. When the gestational age was not known, all stillbirths weighing more than $500 \mathrm{gm}$ were included. These women were examined and relevant investigations done including screening for pre eclampsia, diabetes, thyroid dysfunction, ultrasound reports and other investigations if indicated.

After delivery, the stillborn baby, placenta and umbilical cord were examined.

Examination of stillborn babies included estimation of fetal weight with special note on morphology, skin staining, maceration and colour (pale or plethoric) of the newborn. Placenta was weighed and examined for blood clots, staining with meconium, infarcts, edema, hydropic changes and for structural abnormalities like circumvallate placentae or accessory lobes. Umbilical cord was examined for entanglement, knots (true or false), hematomas, strictures, assessment of Wharton's jelly, number of vessels and insertion abnormalities. Amniotic fluid was noted for volume, colour and odour. Small for gestational age was defined as birth weight below tenth centile for gestational age. Finally the cause found for each stillbirth was classified according to ReCoDe system by Gardosi et al (Annexure 1). ${ }^{3}$
Descriptive statistics (means, standard deviations and percentages) were used for analysis (MS windows version 19, 2010).

\section{RESULTS}

There were 203 stillbirths and 5264 total live births during the study period. 177 cases of stillbirths that fulfilled the inclusion criteria were recruited. Stillbirth rate was 38.56 per 1000 live births.

Table 1: Demographic profile of women with intrauterine fetal deaths.

\begin{tabular}{|l|l|}
\hline Variables & Percentage \\
\hline Rural population & 81.36 \\
\hline Unbooked status & 12.42 \\
\hline Low socioeconomic status & 89.83 \\
\hline BMI $>30$ & 18.64 \\
\hline Age of the mother $>$ 35yrs & 10.73 \\
\hline
\end{tabular}

Table 2: Stillbirths: birth weight, gestational age, maternal age, and parity.

\begin{tabular}{|ll|}
\hline Variables & Mean \pm SD \\
\hline Birth weight in grams & $1311.77 \pm 546.90$ \\
\hline Gestational age in weeks & $30.96 \pm 3.75$ \\
\hline Maternal age in years & $27.28 \pm 4.27$ \\
\hline Parity & $1.68 \pm 0.84$ \\
\hline
\end{tabular}

Most women (89.83\%) were below poverty line (BPL) as per the ration card issued by the state government. (Table 1) A large number $(64.41 \%)$ of them were in the age group of 20 to 29 years. Extremes of age accounted for $4.48 \%$ of cases. Mean maternal age observed was $27.28 \pm 4.27$.

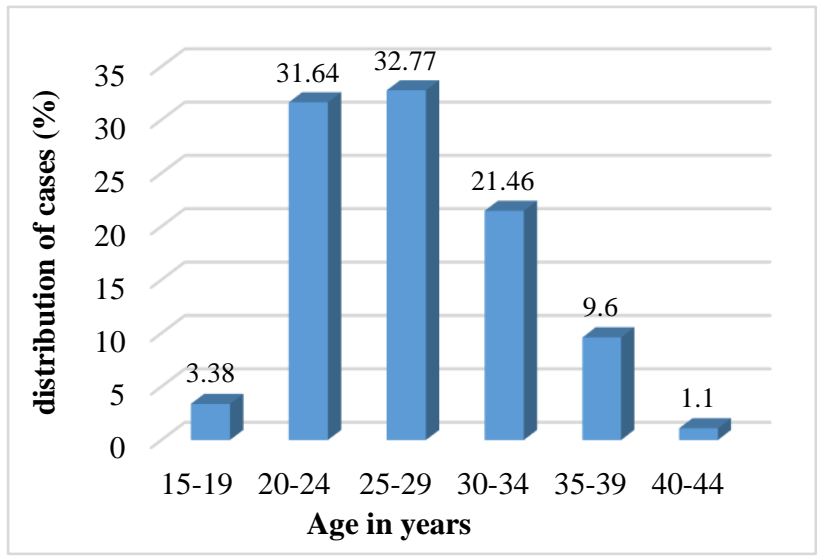

Figure 1: Distribution of still birth according to maternal age.

Stillbirths occurred more often in multi gravidas $(59.32 \%)$ compared to primigravidas $(40.68 \%)$. Mean parity was $1.68 \pm 0.84$. There were 44 cases of previous caesarean section. Seven $(3.95 \%)$ women had a history 
of previous stillbirth. Most women (76.84\%) presented at a gestational age of less than 36 weeks. Mean gestational age was 30.96 \pm 3.75 (Table 2). Among stillbirths 91 were male babies and 86 were female babies. Out of 177 cases $93(52.55 \%)$ were fresh stillborn whereas $84(47.45 \%)$ were macerated at birth.

Table 3 shows the main causes of stillbirths classified according to the ReCoDe system. The ReCoDe system enabled us to explain the cause in $87.58 \%$ of stillbirths. The commonest cause was found to be IUGR $(41.80 \%)$ followed by hypertensive disorders $(27.68 \%)$, congenital anomalies (15.81\%), diabetes (14.68\%), placental abruption (9.03\%), chorioamnionitis (7.91\%) and intrapartum complications (1.12\%). In $12.42 \%$ of cases there was no cause identified (Table 3 ).

Table3: Frequency and percentage distribution according to ReCoDe.

\begin{tabular}{|c|c|c|}
\hline Condition & $\begin{array}{l}\text { Frequency } \\
\text { (n) }\end{array}$ & $\begin{array}{l}\text { Percentage } \\
(\%)\end{array}$ \\
\hline \multicolumn{3}{|l|}{ Group A: foetus } \\
\hline IUGR & 74 & 41.80 \\
\hline Congenital anomaly & 28 & 12.42 \\
\hline Infection & 4 & 2.25 \\
\hline \multicolumn{3}{|c|}{ Group B: Umbilical cord } \\
\hline Prolapse & 5 & 2.82 \\
\hline Constricting loop & 2 & 1.12 \\
\hline \multicolumn{3}{|l|}{ Group C: placental } \\
\hline Abruption & 16 & 9.03 \\
\hline Praevia & 4 & 2.25 \\
\hline \multicolumn{3}{|l|}{ Group D: amniotic fluid } \\
\hline Chorioamnionitis & 14 & 7.91 \\
\hline Polyhydramnios & 4 & 2.25 \\
\hline Oligohydramnios & 41 & 23.16 \\
\hline \multicolumn{3}{|l|}{ Group E: uterus } \\
\hline Rupture & 3 & 1.69 \\
\hline \multicolumn{3}{|l|}{ Group F: mother } \\
\hline Hypertensive disorders & 49 & 27.68 \\
\hline Diabetes mellitus & 26 & 14.68 \\
\hline Jaundice & 1 & 0.565 \\
\hline Thyroid disease & 8 & 4.51 \\
\hline \multicolumn{3}{|l|}{ Group G: intrapartum } \\
\hline Asphyxia & 2 & 1.12 \\
\hline Group H: trauma & Nil & \\
\hline \multicolumn{3}{|l|}{ Group I: unclassified } \\
\hline Unidentified & 22 & 12.42 \\
\hline
\end{tabular}

\section{DISCUSSION}

The ReCoDe system of classification enabled us to identify a cause in $87.58 \%$ of cases of stillbirths. This system of classification has been shown to be effective in explaining the cause of stillbirths in $85 \%$ cases. $^{3}$ The ReCoDe system is among the four classification systems (CODAC, PSANZ-PDC, ReCoDe, and Tulip) which performed well on the Infokeep score. ${ }^{6}$ Among these classification systems, ReCoDe is used exclusively for stillbirths while the others are used for classification of all perinatal deaths.

In present study, $64.41 \%$ of stillbirths occurred in mothers between 20 and 30 years of age (Figure 1). In many earlier studies, maternal age and parity though associated with stillbirth on univariate analysis were not found to be independent risk factors on multivariate analysis. 7However certain other studies have found greater maternal age to be significantly associated with increased risk of stillbirths. ${ }^{8}$

In the present study, $18.64 \%$ of cases had a BMI (Body Mass Index) of more than 30 . Obesity is a modifiable risk factor for stillbirth. Pre pregnancy obesity was found to be associated with a 3.5 fold increased risk for stillbirths after 37 weeks of gestation. ${ }^{9,10}$ The reasons may be placental dysfunction, sleep apnoea, metabolic abnormalities and a difficulty to monitor fetal growth easily.

In present study, $91(51.41 \%)$ were male babies whereas $86(48.58 \%)$ were female babies. Therefore gender was not a significant risk factor for stillbirth. Similar findings have been noted by Sonal et al. ${ }^{7}$ Increased risk of stillbirth associated with male gender has been shown to progressively diminish with increasing birth weight. ${ }^{11}$

There were 44 women (24.88\%) with a history of previous caesarean section. Previous mode of delivery has been shown to influence fetal outcome. Caesarean delivery in a first pregnancy has been associated with an increased risk of stillbirth in the second pregnancy. ${ }^{12}$ Previous history of still birth is a risk factor. In the present study there were 7 cases $(3.95 \%)$ with history of previous stillbirths. Bhattacharya $\mathrm{S}$ et al showed that after adjusting for confounding factors, the odds ratio of recurrence of stillbirths in a second pregnancy was found to be 1.94 (99\% CI $1.29-2.92) .{ }^{13}$ For each stillborn a number of conditions may have contributed to death. Hence a hierarchic grouping system that captures multiple and sequential contributing factors is essential to categorize the causes of fetal death. In ReCoDe systems the hierarchy starts from conditions affecting the fetus, followed by those affecting the umbilical cord, placenta, amniotic fluid, uterus, mother, intrapartum factors and trauma. Each anatomical group is then subdivided into pathophysiological conditions. The primary condition should be first on the list that is applicable to a case of stillbirth. ${ }^{3}$ Fetal growth restriction is included as the last category in group A (denoted as A7). A fetus below $10^{\text {th }}$ centile should be assigned this group only if none of other specific fetal conditions are present. This method of classification thus makes it easy to explain the cause of fetal demise.

Birth weight less than $1 \mathrm{~kg}$ was found in 72 (40.67\%) babies. There were 92 babies $(51.98 \%)$ between $1 \mathrm{~kg}$ and $2.5 \mathrm{~kg}$. Only 13 babies had a birth weight between $2.5 \mathrm{~kg}$ 
and $3.5 \mathrm{~kg}(7.34 \%)$. Birth weight is one of the significant factors for an adverse fetal outcome. Stillbirth is strongly associated with fetal growth restriction. ${ }^{14}$ The risk factors and potential causes of stillbirth and fetal growth restriction mostly overlap. ${ }^{15}$ Other studies have shown a strong relationship between stillbirth and growth retardation with half the stillborns being less than 10 percentile for weight. ${ }^{16}$

Hypertensive disorder of pregnancy was the second most significant condition associated with stillbirth in our study. The overall incidence of stillbirth in this category was $49(27.68 \%)$ (Preeclampsia 29 cases, gestational Hypertension 8, eclampsia 6, HELLP syndrome -6). Similar out comes have been observed in other studies on women with medical complications in pregnancy. ${ }^{17}$ In a WHO antenatal care trial analysis of 39615 pregnancies it was found that fetal deaths were higher in pre-eclampsia $(2.2 \%)$ in comparison to gestational hypertension $(1.4 \%) .^{18}$

Diabetes mellitus was responsible for 26 cases contributing to $14.68 \%$ of stillbirths. Earlier studies have shown the rate of stillbirths to be $1.5 \%$ in diabetic pregnancies. This is five times that of a non-diabetic pregnant population, with majority of the fetal deaths occurring between 34 and 40 gestational weeks.19 Thyroid dysfunction was noted in 8 women; two of whom were hyperthyroid while the other 6 were hypothyroid.

Congenital malformations were present in 28 cases (15.81\%). Most of these were due to open neural tube defects. A very high percentage $(25 \%)$ of stillbirths has been attributed to congenital malformations. ${ }^{20}$ Placental factors also contributed to a significant proportion of intrauterine fetal deaths. In our study 16 cases of stillbirths $(9.03 \%)$ were due to abruptio placentae. Abruptio placentae was associated with adverse perinatal outcomes in previous studies. $^{21}$

There were 14 cases of chorioamnionitis which developed following preterm premature rupture of membranes. One woman had a $\mathrm{CuT}$ in situ. Three women had rupture uterus; two of them with a history of previous Caesarean section and one woman with a history of treatment with methotrexate for invasive mole.

This classification system thus enabled us to explain the cause of fetal death in $155(87.58 \%)$ of the women with intrauterine fetal death. This indeed is an important step towards understanding the causes of still births. If we know what caused an adverse event, necessary changes can be made in the management of future pregnancies to prevent these unfortunate outcomes. The parents and family members can also be counseled based on this information and necessary steps can be taken to avert stillbirths.

\section{Strengths and limitations}

The cross sectional nature of data collection precludes recall bias. Fetal autopsy and radiological assessment was not performed in any of the cases.

\section{CONCLUSION}

The new ReCoDe primary classification system helped us in assigning the probable cause of death in $87.58 \%$ of cases in the study population. Intrauterine growth restriction, which is often a preventable condition constituted the majority of cases. Complete evaluation of cause of death is crucial for counselling the parents for future pregnancies.

\section{Funding: SBMR (State Board for Medical Research) Conflict of interest: None declared \\ Ethical approval: The study was approved by the Institutional Review Board}

\section{REFERENCES}

1. International Statistical classification of diseases and related health problems, $10^{\text {th }}$ revision, Vol. 2 Instruction manual, Geneva World Health Organization; 1993.

2. Lawn JE, Blencowe H, Pattinson R, Cousens S, Kumar R, lbiebele 1. Stillbirths: Where? When? Why? How to make the data count? Lancet. 2011;377:1448-63.

3. Gardosi J, Kady SM, McGeown P. Classification of stillbirth by relevant condition at death (ReCoDe): Population based cohort study. $\mathrm{Br}$ Med J. 2005;331:1113-7.

4. Wigglesworth JS, monitoring perinatal mortality- a pathophysiological approach, Lancet. 1980;31:6847.

5. Aminu M, Unkels R, Mdegela M, Utz B, Adaji S, van den Broek N. Causes of and factors associated with stillbirth in low - and middle-income countries: a systematic literature review. BJOG. 2014;121(4):141-53.

6. Flenady V, Froen JF, Pinar H. An evaluation of classification systems for stillbirth. BMC Pregnancy Childbirth. 2009;9:24.

7. Kumbhare SA, Maitra NK. Aetiological Classification of stillbirths: A Case Control Study. J Obstet Gynecol India. 2016;66(6):420-5.

8. Huang L, Sauve R, Birkett N. Maternal age and risk of stillbirth: a systematic review. CMAJ. 2008; 178:165-72.

9. Nohr EA, Bech BH, Davies MJ. Prepregnancy obesity and fetal death: a study within the Danish National Birth Cohort. Obstet Gynecol. 2005;106:250-9.

10. Stephansson O, Dickman P, Johansson A. Maternal weight, pregnancy weight gain and the risk of antepartum stillbirth. Am J Obstet Gynecol. 2001;184:463-9. 
11. Smith GC. Birth weight and the risk of stillbirth in Scotland 1980-1996. Am J Epidemiol. 2000;151:614-9.

12. Moraitis AA, Oliver-Williams C, Wood AM, Fleming M, Pell JP, Smith GCS. Previous caesarean delivery and the risk of unexplained stillbirth: retrospective cohort study and meta-analysis. BJOG. 2015;122:1467-74.

13. Bhattacharya S, Prescott GJ, Black M. Recurrent risk of stillbirth in a second pregnancy. BJOG. 2010;117(10):1243-7.

14. Mac Dorman MF, Kirmeyer S. Fetal and perinatal mortality United States 2005, Nat/vital stat rep. 2009;57:11-9.

15. Smith GCS, Fretts RC. Stillbirth. Lancet. 2007;370:1715-25.

16. Savvas E, Evangelos A. Case control study of factors associated with intra uterine fetal deaths. Med Gen Med. 2004;6:53.

17. Simpson LL. Maternal medical disease: risk of antepartum fetal death. Semin Perinatol. 2002;26:4250 .
18. Villar J, Carrohil G, Wodyla D. Preeclampsia, gestational hypertension and intrauterine growth restriction related for independent conditions. AJOG. 2006;194:921-31.

19. Persson M, Norman M, Hanson U. Obstetric and perinatal outcomes in diabetic pregnancies: a large population based study. Dia Care. 2009;32:2005-9.

20. Wapner RJ, Lewis D. Genetics and metabolic causes of stillbirth. Semin Perinatol. 2002;26:70-4.

21. Ananth CV, Gethun D, Peltier M R. Placental abruption in term and preterm gestations Obstet Gynecol. 2006;107:785-92.

Cite this article as: Ajini KK, Radha KR, Reena RP. Classification of stillbirths by relevant condition at death $(\mathrm{ReCoDe})$ : a cross sectional study at a rural tertiary care centre in Kerala, India. Int J Reprod Contracept Obstet Gynecol 2017;6:1061-6. 


\section{ANNEXURES}

ANNEXURE I

ReCoDe classification system of etiological classification of stillbirths (relevant condition at death)

Group A: Fetus

Congenital anomaly

Infection

Acute

Chronic

Non immune hydrops

Isoimmunisation

Feto maternal hemorrhage

Twin-twin transfusion

Fetal growth restriction

Group B: Umbilical Cord

Prolapse

Constricting loop or knot

Velamentous insertion

Other

Group C: Placenta

Abruptio

Praevia

Vasa praevia

Other Placental insufficiency

Other

Group D: Amniotic fluid abnormalities

Chorioamnionitis

Poliyhydramnios

Oligohydramnios

Group E: Uterus

Rupture

Uterine anomalies

Other

Group F: Mother

Diabetes

Thyroid disease

Essential Hypertension

Hypertensive diseases in pregnancy

Lupus or antiphospholipid syndrome

Cholestasis

Drug misuse

Others

Group G: Intrapartum

Asphyxia

Birth trauma

Group H: Trauma

External

Iatrogenic

Group I: Unclassified

No relevant condition identified

No information available 